\title{
Cuidado em saúde prestado às mulheres em situação de rua
}

\author{
Health care provided to homeless women
}

\author{
Atención médica brindada a mujeres sin hogar
}

Amanda Pinheiro Magalhães Rocha ${ }^{1}$, Maria Yaná Guimarães Silva Freitas ${ }^{1}$, Vivian Ranyelle Soares de Almeida ${ }^{1 *}$, Diana Cardeal do Nascimento ${ }^{1}$, Jenny Caroline Vieira Moura ${ }^{1}$, Juliana

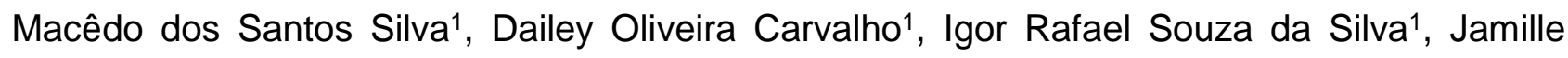
Soares Dias ${ }^{1}$, Geisiane de Almeida Mendonça ${ }^{1}$.

\section{RESUMO}

Objetivo: Descrever o cuidado em saúde prestado às mulheres em situação de rua, pela equipe do Consultório na Rua de uma cidade do interior da Bahia. Métodos: Trata-se de um estudo de natureza qualitativa com caráter descritivo e exploratório, em uma base operacional da equipe do Consultório na Rua em uma cidade do interior da Bahia, local onde os profissionais compartilham as experiências buscando desenvolver da melhor forma suas ações. A coleta de dados foi realizada através de observação passiva, analisada conforme a técnica de Bardin, além de entrevista semiestruturada no período de janeiro a fevereiro de 2020. Resultados: Os profissionais relataram as ações que são desenvolvidas para as mulheres de acordo com o preconizado pelo protocolo do serviço, como: a efetuação de palestras, testes rápidos, vacinação, exames, dentre outros. Foi explanado ainda, sobre a vacinação ser realizada como ação de impacto. Conclusão: A partir do estudo, conclui-se que é necessário um aumento nas estratégias de redução de danos no cuidar de mulheres em situação de rua, ofertando a elas serviço de qualidade, respeito aos seus direitos sexuais e reprodutivos, proteção à saúde mental, fortalecimento do convívio social, orientação sobre direito à alimentação, moradia, segurança, dentre outros.

Palavras-chave: Pessoas em situação de rua, Atenção primária à saúde, Saúde da mulher, Pessoal de saúde.

\section{ABSTRACT}

Objective: To describe the health care provided to homeless women by the staff of the Street Clinic in a city in the interior of Bahia. Methods: This is a qualitative study with a descriptive and exploratory nature, in an operational base of the Consultório na Rua team in a city in the interior of Bahia, where professionals share their experiences seeking to develop their actions in the best way. Data collection was carried out through passive observation, analyzed according to the Bardin technique, in addition to a semi-structured interview from January to February 2020. Results: The professionals reported the actions that are developed for women according to what is recommended by the service protocol, such as: lectures, rapid tests, vaccination, exams, among others. It was also explained about vaccination being carried out as an impact action. Conclusion: From the study, it is concluded that there is a need for an increase in harm reduction strategies in the care of homeless women, offering them quality service, respect for their sexual and reproductive rights, mental health protection, strengthening of social interaction, guidance on the right to food, housing, security, among others.

Keywords: Homeless persons, Primary health care, Women's health, Health personnel.

1 Universidade Estadual de Feira de Santana (UEFS), Feira de Santana - BA.

*E-mail: ranyalmeida98@hotmail.com

SUBMETIDO EM: 2/2022

ACEITO EM: 2/2022

PUBLICADO EM: 2/2022 


\section{RESUMEN}

Objetivo: Describir la atención a la salud proporcionada a las mujeres sin hogar por el personal de la Clínica de la Calle en una ciudad del interior de Bahía. Métodos: Se trata de un estudio cualitativo, de carácter descriptivo y exploratorio, en una base operativa del equipo de Consultório na Rua en una ciudad del interior de Bahía, donde los profesionales comparten sus experiencias buscando desarrollar sus acciones de la mejor manera. La recolección de datos se realizó a través de la observación pasiva, analizada según la técnica de Bardin, además de una entrevista semiestructurada de enero a febrero de 2020. Resultados: Los profesionales informaron las acciones que se desarrollan para las mujeres de acuerdo con lo recomendado por el servicio. protocolo, tales como: charlas, pruebas rápidas, vacunación, exámenes, entre otros. También se explicó que la vacunación se está realizando como una acción de impacto. Conclusión: Del estudio se concluye que existe la necesidad de incrementar las estrategias de reducción de daños en la atención a las mujeres en situación de calle, ofreciéndoles un servicio de calidad, respeto a sus derechos sexuales y reproductivos, protección a la salud mental, fortalecimiento de la interacción social, orientación sobre el derecho a la alimentación, vivienda, seguridad, entre otros.

Palabras clave: Personas sin hogar, Atención primaria de salud, Salud de la mujer, Personal de salud.

\section{INTRODUÇÃO}

O mundo das pessoas em situação de rua é constituído a partir das vivências e experiências vividas tanto individuais, quanto coletivas. A rua é um espaço onde a luta para sobreviver é constante, sendo necessário criar estratégias para ganhar espaço e conseguir se adequar diariamente. As batalhas enfrentadas por essa população são os obstáculos que precisam superar para satisfazer as necessidades básicas enquanto ser humano, criando estratégias para driblar a fome, a violência, os estigmas da sociedade, as condições climáticas e também a falta de assistência à saúde (TULIO R e OLIVEIRA J, 2016).

A experiência de viver no contexto de rua não é uma situação nova, trata-se de uma realidade contemporânea, onde para sobreviver são necessários métodos com a finalidade de se adequar a esse cenário de vulnerabilidade. Nesse sentido, apesar dessa vivência ser geralmente relacionada às péssimas condições econômicas, a falta de emprego e moradia, existem outros fatores que levam pessoas a procurarem as ruas como via de sobrevivência, incluindo as mulheres (SICARI AA e ZANELLA AV, 2018). Esse grupo mesmo em situação de rua merece respeito aos seus direitos sexuais e reprodutivos, a proteção à saúde mental, ao fortalecimento do convívio social, à alimentação, moradia, segurança, entre outros (LACERDA TC, et al., 2017).

Assim, na tentativa de oferecer uma atenção especializada e atender às necessidades de saúde da população em situação de rua, o Ministério da Saúde (MS) através da Portaria no 122, de 25 de janeiro de 2011, definiu as diretrizes do funcionamento e organização das equipes de Consultório na Rua (CnR), atribuindo a mesma, a busca ativa e o cuidado a essa população. Dessa forma, o CnR é um instrumento de saúde integrado a Rede de Atenção Básica e que tem por finalidade dar assistência às pessoas em situação de rua, devendo seguir os princípios e as diretrizes estabelecidas pela Política Nacional de Atenção Básica (PNAB), em consonância com os princípios do Sistema Único de Saúde (SUS) de universalidade, equidade e integralidade, formadas por: enfermeiro, psicólogo, assistente social, terapeuta ocupacional, médico, agente social, técnico ou auxiliar de enfermagem, e técnico em saúde bucal (HALLAIS JAS e BARROS NF, 2015; VARGAS ER e MACERATA I, 2018; MELO EA, et al., 2018).

Quando se trata de mulheres em situação de rua, o cuidado efetivo é baseado no acolhimento e escuta qualificada, visto que esse grupo é socialmente discriminado pelos serviços de saúde; isso pode limitar seu acesso ao atendimento o que frequentemente culmina com o abandono dos acompanhamentos nas Unidades Básicas de Saúde (UBS) seja pelos olhares de reprovação por parte de profissionais e dos clientes, pela indiferença diante de sua condição de saúde ou pelo deslocamento do local de permanência na rua até a unidade (MINISTÉRIO DA SAÚDE, 2012). 
Deste modo, apesar de haver muitos avanços nos direitos sexuais e reprodutivos, as ações nas UBS, em grande parte, seguem uma proposta de atenção materno-infantil, valorizando mais os aspectos biológicos e reprodutivos do que os determinantes sociais de saúde. Isso interfere na vivência de mulheres em situação de rua, já que, em geral, elas têm esses serviços como a única possibilidade de atenção institucional, não sendo resolvida a gênese de problemas macroestruturais (VALLE FAAL, et al., 2020).

Nesse contexto, mesmo com a existência de políticas públicas e estratégias de assistência que viabilizem a garantia de direitos, as mulheres ainda sofrem com o preconceito por estarem em situação de rua; muitos profissionais dos serviços de saúde estão despreparados para lidar com essa população, precisando rever a forma de cuidar e as práticas para esse público feminino mais vulnerável (QUEIROGA RPF, et al., 2018).

A partir do exposto, o interesse em abordar a temática de estudo ocorreu devido a discussões sobre a população em situação de rua e, principalmente sobre a assistência prestada às mulheres nesse cenário em que são amplamente vulneráveis, ficando a margem da sociedade e do governo. Com isso emergiu o objetivo do estudo: Descrever o cuidado em saúde prestado às mulheres em situação de rua, pela equipe do CnR de uma cidade do interior da Bahia.

\section{MÉTODOS}

Trata-se de um estudo de natureza qualitativa com caráter descritivo e exploratório. A escolha desse tipo de estudo foi feita para conhecer de forma mais ampla o fenômeno estudado e a partir daí, delimitar os pontos a serem aprofundados, pois, a pesquisa qualitativa não se preocupa em quantificar dados e nem valores, mas trabalha com os significados dos fenômenos e o aprofundamento da compreensão da realidade de um grupo social ou de uma organização, a fim de entender e interpretar os significados que são atribuídos às suas experiências (TURATO ER, 2005; SOARES SJ, 2019). Diante disso, questiona-se: Como é o cuidado em saúde oferecido às mulheres em situação de rua?

A presente pesquisa foi realizada na base operacional da equipe do $\mathrm{CnR}$ em uma cidade do interior da Bahia, local onde os profissionais se organizam e compartilham as experiências buscando desenvolver da melhor forma suas ações; além de outros lugares, como o centro da cidade, onde as mulheres em situação de rua foram encontradas. Colaboraram com a pesquisa cinco profissionais, cujos critérios de inclusão foram: ser integrante da equipe do $\mathrm{CnR}$; ter prestado serviço à uma (s) mulher (es) em situação de rua há pelo menos 30 dias; e de exclusão: profissionais de saúde em férias e de licença médica.

Inicialmente foi comunicado por meio do parecer substanciado do CEP a autorização da pesquisa ao setor de Educação Permanente da Secretaria Municipal de Saúde e a atenção Primária que é o órgão responsável pela equipe do Consultório na Rua. Posteriormente, foi agendado com a Equipe do Consultório na Rua, através de telefonema, o horário e momento para entrevista e acompanhamento das atividades laborais. $\mathrm{A}$ coordenação da Atenção Básica liberou o documento autorizando a coleta de dados e foi agendado na base operacional da equipe um encontro com a Equipe multiprofissional do Consultório na Rua, local em que a equipe se reúne para programação de atividades/ações de saúde.

Após conhecer a equipe, convidamos os integrantes a participarem da pesquisa e após aceitação, foi feito a apresentação do objeto de estudo e objetivos da pesquisa. Assim, entregamos o Termo de Consentimento Livre e Esclarecido em duas vias para ser lido e assinado pelos participantes da pesquisa. Uma via do termo ficou de posse do (a) entrevistado (a) e a outra da pesquisadora. Foram informados (as) ainda sobre os riscos e benefícios de forma clara e objetiva de acordo com as características individuais de cada participante, e não houve desistência por parte dos entrevistados quanto a participação na pesquisa.

A coleta de dados foi realizada no período de janeiro a fevereiro de 2020 , por meio de entrevista semiestruturada e da observação não participante ou passiva, realizadas, respectivamente, na base operacional da equipe e nos espaços urbanos. Foram empregados como instrumentos de coleta de dados: $\mathrm{O}$ roteiro de entrevista semiestruturado composta por dois blocos de informações que abordaram características sociodemográficas dos participantes, do objeto investigado; e de observação não participante. 
Para análise dos dados utilizou-se a técnica de análise de conteúdo, constituída em três etapas: préanálise, exploração do material e tratamento dos resultados - inferência e interpretação. A primeira fase (préanálise) configurou-se como a etapa da organização do material, havendo o primeiro contato com documentos, envolvendo uma leitura "flutuante" para formulação de hipóteses. Nessa fase, foram executadas as entrevistas e transcritas, possibilitando melhor entendimentos dos futuros resultados (BARDIN L, 2016).

A segunda fase, ou fase de exploração do material, consistiu na estruturação das informações adquiridas, a partir da escolha das unidades de codificação. A terceira fase (tratamento dos resultados - inferência e interpretação) e última, destinou-se ao tratamento dos resultados, tornando-os significativos e válidos. Essa interpretação foi realizada de forma a transcender o conteúdo encontrado nos documentos com inferências a fim de adiantar interpretações.

Esta técnica foi apropriada uma vez que permitiu a interação entre a pesquisadora e os participantes do estudo, com o intuito de angariar dados acerca da experiência dos profissionais de saúde diante de suas vivências quanto ao cuidado às mulheres em situação de rua, de forma aprofundada. Além disso, a observação do trabalho do (a) profissional do Consultório de Rua não causou interferência na rotina dos serviços de assistência à saúde das mulheres, nem interviu no andamento das consultas ou ações de saúde.

O estudo acatou a Resolução $n^{\circ} 466$, de 12 de dezembro de 2012, sobre pesquisa envolvendo seres humanos considerando os princípios de autonomia, beneficência, não maleficência e justiça e a Resolução no 510, de 07 de abril de 2016 do Conselho Nacional de Saúde, que regulamenta pesquisas que envolvem seres humanos no Brasil (BRASIL, 2012; RASIL, 2016), estando regulamentado no Comitê de Ética em Pesquisa (CEP) com parecer satisfatório de número 3.747.208.

Salienta-se que todas as informações foram mantidas em confidencialidade e sem divulgação dos nomes dos participantes, respeitando, assim, a privacidade e os princípios éticos da pesquisa. Para isso, as entrevistas foram codificadas por numeração de modo que o sigilo, o nome e a identidade profissional ficaram preservadas. Assim, utilizou-se a combinação da letra inicial da palavra entrevistado (E), seguida de um número, conforme exemplo a seguir: E1, E2, E3, e assim sucessivamente.

\section{RESULTADOS E DISCUSSÃO}

A pesquisa procedeu com a entrevista de 5 (cinco) profissionais de saúde que integram a equipe do $\mathrm{CnR}$. As características dos participantes foram: idade média de 40 anos (variação de 32 a 53 anos); $60 \%$ eram do sexo feminino, a escolaridade mais frequente foi nível superior completo; e o tempo de atuação no programa CnR foi de 4 a 5 anos.

\section{Atividades desenvolvidas pela equipe do $\mathrm{CnR}$}

Os profissionais relataram as ações que são desenvolvidas para as mulheres de acordo com o preconizado pelo protocolo do serviço, como: a efetuação de palestras, testes rápidos, vacinação, exames, dentre outros. Foi explanado ainda, sobre a vacinação ser realizada como ação de impacto, garantindo a imunização de adolescentes, das mulheres em idade fértil e as grávidas:

"A gente faz palestra sobre orientação sexual, preservativos, DST, faz teste rápido, a gente faz vacinação, a gente acompanha as que são gestantes também, o prénatal [...]" (E1).

"Palestras, distribuição de preservativo, exames, teste rápido é feito pela colega enfermeira [...]" (E2).

"Os testes rápidos nós realizamos na rua com frequência, é até uma coisa que é muito importante dos pacientes, eles nos procuram muito para realizar o teste rápido e isso é muito positivo, e a gente estimula com eles uma periodicidade de a cada três meses a gente tá realizando, repetindo os exames, né, também pela questão da vulnerabilidade que eles se encontram" (E3). 
"A gente faz a distribuição dos preservativos, né? O que não é muito assim, digamos $100 \%$, porque na hora que eles estão sob efeito de droga, não vão lembrar de preservativo, né?" (P4).

"[...] ações do consultório na rua são iguais às ações no Programa de Saúde da Família PSF, só que a gente não tem o muro, não tem a casa, mas a gente faz a escuta, a gente examina, a gente pede exames, a gente prescreve, a gente acompanha" (E5).

"Nós realizamos a imunização, de forma como ação de impacto, não de ação eletiva" (E3).

A Portaria ํํ 122 de 25 de janeiro de 2012 dispõe sobre as diretrizes de organização e funcionamento das equipes do Consultório de Rua, no qual é uma equipe que integra o componente da Rede de Atenção Psicossocial e que desenvolve ações de Atenção Básica e por isso deve prestar assistência de acordo com os fundamentos da Política Nacional de Atenção Básica, visando ações de promoção, prevenção, proteção, tratamento, redução de danos, que deve ser efetuada por uma equipe multiprofissional (BRASIL, 2012).

Os profissionais expuseram que as ações prestadas são realizadas por meio da educação em saúde voltada para a redução de danos, como ferramenta de ensino sobre planejamento familiar, prevenção de Infeções Sexualmente Transmissíveis (IST) (CONCEIÇÃO DS, et al., 2020). A educação em saúde é o processo de aprendizagem que perpassa a transmissão do ensino, pois o saber é compartilhado e a construção do conhecimento se dá pelo estabelecimento de um diálogo entre educador (equipe) e educando (mulher em situação de rua), configurando uma educação problematizadora, tornam-se sujeitos de processo de crescimento e aprendizado mútuo (ALECRIM TFA, et al., 2016).

No Brasil, foram notificados 15.923 casos de infecção por Vírus da Imunodeficiência Humana (HIV) no ano de 2019 (MINISTÉRIO DA SAÚDE, 2019). Isso mostra a importância de estar informando e orientando quanto às formas de prevenção de IST e da Síndrome da Imunodeficiência Adquirida (AIDS), além da necessidade de periodicidade de realização de testes rápidos, para detecção ativa da doença (MONTEIRO SS, et al., 2019). De acordo com os resultados encontrados na pesquisa em questão, os testes rápidos são executados pela equipe com frequência e a procura do procedimento é feito pelas próprias mulheres na maioria das vezes, além do teste rápido são distribuídos preservativos e realizada a orientação do seu uso.

A respeito da imunização, relatada pelos profissionais, é uma prática exercida pela equipe em consonância com a Secretaria de Saúde, que fornece os recursos e insumos necessários para que a equipe realize a vacinação como ação de impacto, podendo ser feito durante as campanhas proporcionadas por esse serviço. Corroborando com esses achados, uma pesquisa revela que as vacinas são desenvolvidas a partir de bactérias e vírus atenuados ou mortos que em contato com o organismo humano geram reações de memória imunológica que perante uma nova invasão produzirá anticorpos específicos garantindo assim uma resposta imune eficaz impedindo que o indivíduo adoeça (NASSARALLA APA, et al., 2019).

O pré-natal das gestantes é realizado na rua pela equipe do $\mathrm{CnR}$ como informado pelos profissionais durante a entrevista. Estudos asseguram que essas consultas, quando prestada com qualidade, podem reduzir os riscos e agravos durante o parto (GUIMARÃES EAA, et al., 2017). A realização do acompanhamento permite identificar os fatores de risco que trazem complicações à gestação, além de permitir a detecção precoce e tratamento adequado, contribuindo para um desfecho materno e perinatal favoráveis (ARAUJO AS, et al., 2017).

Sendo assim, a assistência adequada pode partir de atividades educativas, proporcionando respostas diante dos questionamentos da mulher, levando informações necessárias sobre a gestação e os cuidados com o recém-nascido; a realização de testes rápidos, de exames laboratoriais e ultrassonografia nos serviços intersetoriais, buscando ofertar o melhor cuidado perante o que é disponibilizado para o desenvolvimento do trabalho (ROCHA RS e SILVA MGC, 2012; MACHADO MPM e RABELLO et al., 2018). 


\section{Encaminhamento das demandas}

Com relação ao encaminhamento, os profissionais informaram quanto à necessidade diante de alguma demanda que não conseguem resolver, seja por falta de recursos ou pela necessidade de um serviço de média ou alta complexidade:

"[...] encaminhamos para exames no laboratório do hospital da mulher, temos 5 vagas por dia, são realizamos todos os exames. Centro Municipal de Diagnóstico e Imagem (CMDI), encaminhamos para ultrassom, e assim, a gente vai passando no médico [...]" (E2).

"A própria portaria do serviço do consultório na rua, ela diz que essas mulheres ou são encaminhadas às unidades básicas de saúde, porque uma vez que nós não temos condições de realizar os exames de preventivo, mamografia, transvaginal, então elas são encaminhadas aos serviços de referência" (E3).

"[...] o consultório trabalha com saúde, é uma equipe de saúde que trabalha com a saúde. Saúde física, saúde mental. Saúde psíquica. Então a necessidade dela é um encaminhamento? Você vai encaminhar. Esse é o trabalho. É a demanda. Vai de acordo a demanda, entendeu?" (E4).

Por não ter uma unidade fixa para a assistência às mulheres, a equipe do $\mathrm{CnR}$ tem o processo de trabalho ainda mais desafiador, uma vez que os profissionais que vão ao encontro daquelas mulheres para execução do atendimento, dificultando a garantia da oferta de todos os recursos necessários para a demanda da mulher, e principalmente a realização do número de consultas preconizado pelo MS nos diversos programas direcionados a esse público. Neste contexto, a partir do problema enfrentado pela mulher, a equipe identifica a demanda e, caso haja necessidade, realiza o encaminhamento para o PSF ou para o serviço de média ou alta complexidade, como hospitais e clínicas de imagem.

Para os profissionais, o acesso a estes dispositivos possibilita maiores chances de resolubilidade das necessidades desses usuários. Entretanto, o encaminhamento não é garantia de mulher em situação de rua frequentar a unidade de saúde, seja por sentir-se rejeitada pela equipe de saúde ou pela clientela da unidade de saúde, além de não priorizar o cuidado consigo.

Um estudo realizado com a população de rua na cidade de Calgary no Canadá revelou barreiras que impedem o acesso aos serviços de saúde por essa população, dentre elas os sentimentos de vergonha e baixa autoestima; outro fator foi de que as mulheres estivessem menos dispostas a ir a uma clínica localizada numa área em que não estão familiarizadas ou se sentirem desconfortáveis diante do potencial de vigilância e controle público e/ou policial (CAMPBELL DJT, et al., 2015). Esse achado valida o resultado desse estudo, uma vez que as pessoas em situação de rua vivem muitos dilemas que são carregados de preconceitos, medos, angústias, discriminação por parte dos profissionais de saúde e da clientela do serviço, configurando na desistência do atendimento.

A resolubilidade nos serviços de saúde se configura em dar uma resposta às demandas de saúde da mulher de acordo com as necessidades naquele momento, de forma a garantir o acesso ao atendimento (CARDOSO AC, et al., 2018). Vale salientar que os agendamentos necessitam ser marcados pela equipe do $\mathrm{CnR}$, e os mesmos devem executar supervisão sistemática para que os exames sejam realizados e a paciente reavaliada.

\section{CONCLUSÃO}

A partir do estudo, conclui-se que é necessário um aumento nas estratégias de redução de danos no cuidar de mulheres em situação de rua, ofertando a elas serviço de qualidade, respeito aos seus direitos sexuais e reprodutivos, proteção à saúde mental, fortalecimento do convívio social, orientação sobre direito à alimentação, moradia, segurança, dentre outros. Dessa forma, tornam-se imprescindíveis políticas públicas para facilitar a adesão das mulheres em situação de rua aos serviços de saúde, analisando cada uma de acordo com sua particularidade. 


\section{REFERÊNCIAS}

1. ALECRIM TFA, et al. Experience of health professionals in care of the homeless population with tuberculosis. Revista da Escola de Enfermagem da USP, 2016; 50(5): 809-816.

2. ARAUJO AS, et al. contexto da gestante na situação de rua e vulnerabilidade: seu olhar sobre o pré-natal. Revista de enfermagem UFPE online, 2017; 11(Supl. 10): 4103-4110.

3. BARDIN L. Análise de conteúdo. São Paulo: Edições 70, 2016.

4. BRASIL. Conselho Nacional de Saúde. Resolução no 466. Diário Oficial da União. 2012. Disponível em: https://conselho.saude.gov.br/resolucoes/2012/Reso466.pdf. Acessado em: 20 de novembro de 2021.

5. BRASIL. Conselho Nacional de Saúde. Resolução no 510. Diário Oficial da União. 2016. Disponível em: http://conselho.saude.gov.br/resolucoes/2016/Res0510.pdf. Acessado em: 14 de outubro de 2021.

6. CAMPBELL DJT, et al. Primary healthcare needs and barriers to care among Calgary's homeless populations. BMC Family Practice, 2015; 16(139): 1-10.

7. CARDOSO AC, et al. Challenges and potentialities of nursing work in street medical offices. Revista Latino-Americana de Enfermagem, 2018; 26:3045.

8. CONCEIÇÃO DS, et al. A educação em saúde como instrumento de mudança social. Brazilian Journal of Development, 2020; 6(8): 59412-59416.

9. GUIMARÃES EAA, et al. Prematurity and associated factors in Divinópolis, Minas Gerais state, Brazil, 2008-2011: analysis of the Information System on Live Births. Epidemiologia e Serviços de Saúde, 2017; 26(1): 91-98.

10. HALLAIS JAS, BARROS NF. Consultório na Rua: visibilidades, invisibilidades e hipervisibilidade. Caderno de Saúde Pública, 2015; 31(7): 1497-1504.

11. LACERDA TC, et al. Tuberculosis infection among primary health care workers. Jornal Brasileiro de Pneumologia, 2017; 43(5): 416-423.

12. MACHADO MPM, RABELLO ET. Competências para o trabalho nos Consultórios na Rua. Physis: Revista de Saúde Coletiva, 2018; 28(4): 280413.

13. MELO EA, et al. Mudanças na Política Nacional de Atenção Básica: entre retrocessos e desafios. Saúde em Debate, 2018; 42(1): 38-51.

14. MINISTÉRIO DA SAÚDE. Portaria $\mathrm{n}^{0} \quad 122 . \quad 2011 . \quad$ Disponível em: https://bvsms.saude.gov.br/bvs/saudelegis/gm/2012/prt0122_25_01_2012.html. Acessado em: 4 de novembro de 2021.

15. MINISTÉRIO DA SAÚDE. Boletim Epidemiológico HIV/Aids. 2019; 1: 72. Disponível em: http://www.aids.gov.br/ptbr/pub/2019/boletim-epidemiologico-de-hivaids-2019. Acessado em: 4 de novembro de 2021.

16. MONTEIRO SS, et al. Challenges facing HIV treatment as prevention in Brazil: an analysis drawing on literature on testing. Ciência \& Saúde Coletiva, 2019; 24(5): 1793-1807.

17. NASSARALLA APA, et al. Dimensões e consequências do movimento antivacina na realidade brasileira. Revista Educação Saúde, 2019; 7(Supl. 1): 120-125.

18. QUEIROGA RPF, et al. Tuberculosis in the homeless population: performance of primary health care professionals. Revista René, 2018; 19: 32463.

19. ROCHA RS, SILVA MGC. Assistência pré-natal na rede básica de fortaleza-CE: uma avaliação da estrutura, do processo e do resultado. Revista Brasileira em Promoção da Saúde, 2012; 25(3): 344-355.

20. SICARI AA, ZANELLA AV. Pessoas em Situação de Rua no Brasil: Revisão Sistemática. Psicologia: ciência e profissão, 2018; 38(4): 662-679.

21. SOARES SJ. Pesquisa científica: uma abordagem sobre o método qualitativo. Revista Ciranda, $2019 ; 1$ (3): $168-180$.

22. TULIO R, OLIVEIRA J. Attention and health care of the people living on the streets. Psicologia em Estudo, 2016; 21(1): 101-113.

23. TURATO ER. Qualitative and quantitative methods in health: definitions, differences and research subjects. Revista Saúde Pública, 2005; 39(3): 507-514.

24. VALLE FAAL, et al. Health-interfering streets experiences: homeless people's perspective. Saúde Debate, $2020 ; 44$ (124): 182-192.

25. VARGAS ER, MACERATA I. Contributions of Street Outreach teams to primary health care and management. Revista Panam Salud Publica, 2018; 42: 170. 